\title{
SciComm Report, Hacia la Sistematización de la Comunicación de las Ciencias
}

\section{SciComm Report, Towards the Systematization of Science Communication}

\author{
IVÁN SUAZO (1) \\ Universidad Autónoma de Chile, Chile. \\ VANIA FIgUEROA (1) \\ Centro de Comunicación de las Ciencias, Universidad Autónoma de Chile, Chile. \\ Juan Carlos Beamín (i) \\ Centro de Comunicación de las Ciencias, Universidad Autónoma de Chile, Chile. \\ Autor de correspondencia: Dr. Iván Suazo Galdames. \\ Correo: scicomm@uautonoma.cl \\ Vicerrectoría de Investigación y Postgrado, Universidad Autónoma de Chile
}

La circulación del conocimiento en la sociedad fue por muchos años un proceso lento y lineal, en el cual la producción realizada por la comunidad científica era acumulada enciclopédicamente en libros cuyo acceso era limitado a la comunidad general. Con el desarrollo de las revistas científicas especializadas la ciencia constituyó verdaderos guetos del conocimiento aceptado por las comunidades expertas, denominados paradigmas (Kuhn, 1970). Esta forma de transferencia del conocimiento ha sido considerado la raíz del denominado conflicto social sobre la ciencia, el cual debía ser resuelto mediante la educación formal, a través de un proceso de alfabetización científica (Nisbet and Scheufele, 2009). La historia muestra que este modelo no contribuyó significativamente a mejorar los niveles de cultura científica en la sociedad, como ejemplo, durante la primera década del siglo XXI se duplicó la cantidad de estadounidenses que accedió a la Universidad y la información científica creció exponencialmente en la Web, no obstante las encuestas nacionales que miden conocimiento científico no variaron significativamente ("S\&E Indicators 2008 ” n.d.). 
Por otra parte, existe una valoración de la ciencia en la sociedad, según un estudio del Pew Research Center for the People \& the Press el $70 \%$ de las personas encuestadas consideraba que las y los científicos contribuyen mucho a la sociedad ("Public Praises Science; Scientists Fault Public, Media | Pew Research Center” n.d.), en concordancia, el estudio más grande del mundo sobre percepción social de la ciencia señala que el $72 \%$ de las personas a nivel mundial confía en las y los científicos, pero más de la mitad (57\%) de la población mundial cree que no sabe mucho, si es que sabe algo, sobre ciencia (Gallup, 2019).

En los últimos años las personas han estado expuestas a grandes cantidades de información, el conocimiento está disponible, permitiendo que éste circule de diversas formas en la sociedad. También se han conocido diversas formas de malas prácticas que antes se manejaban en círculos reducidos ("Science, Now Under Scrutiny Itself - The New York Times" n.d.).

Por otra parte, la construcción de buenas políticas públicas requiere de ciudadanos con conocimientos de la evidencia sobre la ciencia y la tecnología involucradas. Sobre muchos temas de actualidad (cultivos transgénicos, vacunas, cambio climático), sectores del público tienen opiniones diferentes a las de la mayoría de las y los científicos y, a menudo, no apoyan propuestas basadas en el consenso científico (Fahnestock, 2020)vaccinations, climate change, al respecto Carl Sagan (1997) afirmaba hace más de dos décadas que "La ciencia por sí sola no puede defender cursos de acción humana, pero ciertamente puede iluminar las posibles consecuencias de cursos de acción alternativos."(p27)

La ciencia debe ser comunicada, este es un rol ineludible de las personas dedicadas a ella, de las instituciones y del Estado. En 1898, Santiago Ramón y Cajal sentenciaba que la posteridad duradera de las naciones es obra de la ciencia (Cajal, n.d.), esta frase vigente aun, debe acompañarse de la obligación del Estado de promover sociedades más libres a través del acceso al conocimiento.

Aunque la comunicación científica se entiende típicamente en términos de divulgación de la investigación científica al público en aras de la alfabetización científica, una mirada rápida a las métricas alternativas (Altmetrics) nos muestra que el conocimiento circula por distintas vías y contribuye a formación de opinión, más allá de los círculos expertos (Soós and Kiss 2020). Es este impacto social de la ciencia el que debe ser estudiado a partir de las diversas formas que adopta la comunicación científica y la divulgación.

La comunicación sistemática de la ciencia y del conocimiento debe ser evaluada, debemos reflexionar acerca de sus prácticas y medir su impacto en la sociedad, de ahí la necesidad de la creación de la Sci Comm Report, que siguiendo los pasos de otros referentes como el Journal of Science Communication y Science Communication, buscan servir de espacio de diálogo interdisciplinario para todos los que comunican ciencia.

La SciComm Report surge como una publicación de acceso abierto, revisada por pares interesada en artículos que buscan comprender cómo circula el conocimiento en la sociedad en todas sus formas. La revista cubre una amplia variedad de temas relacionados con la comunicación de las ciencias y el conocimiento, desde las disciplinas STEM y también desde las ciencias sociales y humanidades. Son bienvenidos los artículos que abordan objetivos de transferencia 
de los resultados a la comunidad, derivados de investigación convencional, investigaciones relacionadas al impacto de experiencias en divulgación y comunicación de las ciencias. También el reporte de experiencias sistemáticas de ciencia ciudadana, así como la comunicación ambiental y de salud.

Esperamos que la SciComm Report sea una contribución a la profesionalización de la comunicación científica en Iberoamérica.

\section{REFERENCIAS}

Cajal, Santiago Ramón y. n.d. "CVC. Santiago Ramón y Cajal. Reglas y Consejos Sobre Investigación Científica.” 1898.

Fahnestock, Jeanne. "Rhetorical Citizenship and the Science of Science Communication." Argumentation 34 (3) 2020 : 371-87. https://doi. org/10.1007/s 10503-019-09499-7.

Gallup. 2019. Wellcome Global Monitor- First Wave Findings. Accessed October 13, 2020. https://wellcome.org/sites/default/files/ wellcome-global-monitor-2018.pdf

Kuhn, Thomas S. 1970. The Structure of Scientific Revolutions Second Edition, Enlarged. International Encyclopedia of Unified Science.

Nisbet, Matthew C., and Dietram A. Scheufele. "What's next for Science Communication? Promising Directions and Lingering Distractions." American Fournal of Botany 96 (10) 2009: 1767-78. https://doi.org/10.3732/ajb.0900041.

"Public Praises Science; Scientists Fault Public, Media | Pew Research Center." n.d. Accessed October 11, 2020. https://www.pewresearch. org/politics/2009/07/09/public-praisesscience-scientists-fault-public-media/.

Sagan, C. The demon-haunted world: science as a candle in the dark. 1st Ballantine Books ed. New York: Ballantine Books, 1997.

"S\&E Indicators 2008." n.d. Accessed October 11, 2020. https://wayback.archive-it. org/5902/20160210152939/http://www.nsf. gov/statistics/seind08/.

"Science, Now Under Scrutiny Itself - The New York Times." n.d. Accessed October 11, 2020. https://www.nytimes.com/2015/06/16/ science/retractions-coming-out-from-underscience-rug.html.

Soós, Sándor, and Anna Kiss. "Informetrics and the Study of Science-Society Communications: A Bibliometric Scoping Review." Scientometrics 124 (2) 2020: 825-42. https://doi.org/10.1007/ s $11192-020-03444-2$. 